\title{
Spectrum of Mycotic corneal ulcers in \\ Mid Western peripheral region of Terrain belt of Nepal and Indo-Nepal Border
}

\author{
Bastola $\mathrm{P}^{1^{*}}$ Mishra $A,{ }^{2}$ Chaudhary $N,{ }^{2}$ Nath $H K,{ }^{2}$ Mehrotra $A N^{2}$
}

${ }^{1,2}$ Nepalgunj Eye Hospital, ${ }^{2}$ Nepalgunj Medical Collge and Teaching Hospital, Kohalpur, Nepalgunj, Nepal

\author{
*Corresponding Author: \\ Dr. Pradeep Bastola, MD \\ Ophthalmologist, \\ Femtosecond laser assisted cataract surgeon, \\ Oculoplastic surgeon, \\ Assistant professor, Nepalgunj Medical College, Nepalgunj \\ Medical Director, Nepalgunj Eye Hospital, Nepalgunj, Nepal. \\ Email:pbs_dinku@yahoo.com
}

\section{Citation}

Bastola P, Mishra A, Chaudhary N, Nath HK, Mehrotra AN. Spectrum of Mycotic corneal ulcers in Mid Western peripheral region of Terrain belt of Nepal and IndoNepal Border. Nepal Journal of Medical Sciences 2013;2(1):42-7.

\begin{abstract}
Background: The study was set in two different tertiary eye care hospitals of Nepal. The study area covered a large population of mid western, far western peripheral terrain regions of Nepal as well as the corresponding Indo-Nepal border. Looking at the high prevalence of mycotic corneal ulcers in the region, the study aimed to find out the pattern of culture proven fungal corneal isolates in diagnosed cases of mycotic corneal ulcers.
\end{abstract}

Methods: A prospective hospital based study was carried out on 2768 consecutive patients presenting with corneal ulcer in the outpatient department of Ophthalmology in Nepalgunj Medical College and Nepalgunj Eye Hospital, Nepalgunj Nepal from July, 2011 to July, 2012. Patient particulars with regard to the socio demographic data, predisposing risk factors, prior treatment modalities, laboratory results and the distribution pattern of culture proven fungal isolates were analyzed.

Results: Diagnosis of various types of pure fungal keratitis was established post investigations in $760(27.45 \%)$ of all patients. Men 444 $(58.42 \%)$ were more commonly affected than women. The young adults age group of 31-40 years was most commonly involved $220(28.94 \%)$ in number. Trauma or injury to cornea $431(56.71 \%)$ was the commonest predisposing risk factor. Fusarium species (Fusarium spp.) were the commonest fungal isolates accounting for 287 (37.76\%) of diagnosed fungal ulcer cases, followed by curvularia 136 (17.89\%).

Conclusion: Fusarium species are the commonest fungal isolates in cases of fungal corneal ulcer in mid western peripheral terrain belt of Nepal followed by curvularia and unidentified dematiaceous fungi.

Keywords: Corneal ulcer; fungal isolates; fusarium species; cornea; mycotic fungal corneal ulcers

\section{Background:}

C and intervention as it may lead to irreversible damage to the cornea in short span of time and consequently blinding eye disease. Suppurative (bacterial and mycotic) corneal ulcer is still one of the leading causes of blindness. In Nepal annual incidence is 799 cases per 1,000,000 population, of which nearly $22 \%$ are of fungal origin. ${ }^{1}$

Corneal ulcer, trauma to the eyes and sequale of corneal ulcer especially corneal scarring/corneal opacity stands only second to cataract worldwide, as the commonest cause of blindness in any age group. Various studies have concluded; 
it is very common in the developing and underdeveloped nations. Hereby showing the importance of prevention, early diagnosis and treatment of suppurative corneal ulcers cases. $^{1-6}$

Previously, it was thought various ocular pathologies like trachoma, neonatal Ophthalmia, xerophthalmia and onchocerciasis (in Africa) play big role in developing corneal scarring or corneal opacity added by harmful eye practices by patients, medications by village healers and lack of specialist medical personnel. However, all recent studies and conclusions have made it clear that primary corneal ulceration is the major cause of corneal scarring or opacity. $3,7,8,9$

Actual data on the annual incidence of infective ulceration are difficult to obtain in most countries. Available data indicate that, in the United States there are 11 corneal ulcers per 100,000 population annually. ${ }^{10}$ In southern part of India the number is 10 times higher with 113 per 100,000 population per year. ${ }^{11}$ Corneal injury with vegetative matters highly increases the risk of developing fungal corneal ulcers. However, the culture proven fungal isolates can differ in different geographical areas. ${ }^{12,13}$

As the recent studies or survey done in Nepal have been pointing towards changing pattern of ocular morbidity. ${ }^{14}$ This study was an attempt to find out and gather valuable information related to common types of pure fungal isolates in cases of diagnosed fungal corneal ulcers in mid western, far western peripheral terrain region of Nepal and corresponding areas of Indo-Nepal border. So that it helps in providing concrete evidence, in diagnosing and treating the cases more accurately.

\section{Methods:}

A hospital based, prospective, observational study was carried out with a primary objective to find out the distribution pattern of the common fungi in cases of fungal corneal ulcer from July, 2011 to July, 2012. All corneal ulcer cases attending the outpatient department and cornea clinic of two of the tertiary eye hospitals of mid western peripheral terrain belt of Nepal, (Nepalgunj Eye Hospital and Nepalgunj Medical College and Teaching Hospital, Nepalgunj) were included in the study. An informed consent was taken from all the study subjects. The study strongly adhered to the Tenets of the Declaration of Helsinki and also was approved by the ethical research committee of Nepalgunj Eye Hospital, Nepalgunj. Both the tertiary eye hospitals are well equipped with modern laboratory investigations.
The cases which were clinically diagnosed as a typical viral corneal ulcer, healing corneal ulcers, cases with mixed infections, non suppurative corneal ulcers, cases who have been already undergone investigations and were on treatment for corneal ulcer on presentation and cases not willing to be the part of study were excluded from the study.

A total of 2768 cases, after exclusion based on the above specified criteria, were evaluated in the outpatient department and cornea clinic over a period of one year. Corneal ulcer was defined as loss of corneal epithelium with variable corneal stromal infiltration, with clinical evidence of infection with or without hypopyon. A detailed history taking including systemic illnesses, clinical examination including ocular/slit-lamp bio-microscopic examination of the anterior segment of the eye with more focus to the corneal ulcer was carried out; when needed and when visible fundus evaluation was carried out in affected eye while routine detailed ocular examination was carried out in normal eye. In the affected eye after staining the ulcer with sodium fluroscein the size of the ulcer, stromal infiltrate and depth was measured using the variable slit on the slit lamp and recorded in millimeter. The hypopyon if present was noted and measured similarly in millimeters. The ulcer margin, thinning of the floor, satellite lesions, any retained foreign body and pigmentation over the ulcer surface was recorded. A diagram of each ulcer was drawn on the standardized form by performing frontal and cross sectional sketches. Associated ocular conditions like blepharitis, conjunctivitis, dacryocystitis, corneal anesthesia, dry eyes, lid abnormalities, lagophthalmos, past surgery in the cornea, use of contact lens and corticosteroids were noted.

One corneal swab and three corneal scrapings were collected from each patient by an Ophthalmologist with all aseptic precautions to detect or to exclude possible bacterial corneal ulcer or mixed growth. Corneal swab was taken by rubbing the ulcerated area of the cornea with sterile cotton swab soaked with sterile normal saline before instillation of local anesthetic and was used for Gram, Giemsa staining purpose. ${ }^{15}$ For taking corneal scrapings; two drops of preservative free local anesthetic ( $1 \%$ Proparacaine) were put in the affected eye. Five minutes after instillation of local anesthetic, three corneal scrapings were taken by sterile Bard Parker No. 15 scalpel blade or a flame sterilized platinum Kimura spatula under a biomicroscope. Great care was taken for not to touch the lashes or lids and to obtain material from the base and the peripheral margins of ulcer. The swab was inoculated onto Blood agar, MacConkey's agar, and Chocolate agar media and incubated aerobically at 37 degrees Celsius for 
maximum up to 48 hours. To ensure 5-10\% CO2, incubated Chocolate agar plates were put under candle extinction jar. All the bacterial isolates were identified by their colony morphology, gram staining, motility testing by hanging drop preparation, pigment production and relevant biochemical tests. ${ }^{16,17}$

To detect fungal corneal ulcers, corneal scrapings were used for wet preparation in $10 \% \mathrm{KOH}$, Gram stain and Giemsa stain. For fungus culture; materials obtained by scraping were spot inoculated on plain Saboraud's dextrose agar medium (SDA) and in to Brain Heart Infusion Broth (BHI).

The inoculation technique consisted of "C" streaks on the culture plate, with the idea to localize the site of implantation of the corneal scraping on the agar media. Inoculated SDA media was incubated at 25 degree Celsius. Only growth occurring on the "C" streaks was considered as significant and out growth away from the " $\mathrm{C}$ " streak was discarded as contaminants. ${ }^{18}$

The plates which did not show any evidence of growth after 14 days were discarded. For identification of fungal species that grown in SDA, microscopical examination in wet preparation was done besides subculturing onto SDA media.

Any growth observed was identified using standard bacteriological and mycological techniques. ${ }^{19}$ Microbial cultures were considered significant if any/all of the following criteria were satisfied: (1) growth of the same organism was demonstrated on more than one solid medium; (2) confluent growth was seen at the site of inoculation on one solid medium; (3) growth of one medium was consistent with direct microscopy findings (appropriate staining and morphology with Gram stain); (4) the same organism was grown from repeated scraping material. ${ }^{20}$

Cultures were considered negative if no growth was found after even 96 hours. Identification of fungi in SDA medium was done by a trained microbiologist and a group of ophthalmologists. For all cases, treatment was commenced empirically with broad spectrum topical antibiotics immediately after the diagnosis was made, most of the cases were admitted, while the cases refusing admission were followed up daily with same medications. Subsequent treatment was tailored according to the microbiological diagnosis and sensitivity results. The final visual acuity was defined as the visual acuity on discharge from the ward. The patients were asked to follow-up every 7 days for 3 weeks and then 2 weekly for 2 months, after which follow-up was individualized as per patient's requirements. A careful examination of the staining pattern and culture growth with the help from the microbiologist made sure correct data and information were entered in the proforma specifically designed for the study. Positive findings were recorded and data was entered in the statistical package for social sciences (SPSS) data base version 19.0 and the results were interpreted.

\section{Results:}

A total of 2768 consecutive cases meeting inclusion criteria were included in the study. Out of the total study population $760(27.45 \%)$ cases were pure fungal isolates. Men 444 $(58.42 \%)$ were more commonly affected than women 316 $(41.48 \%)$. The commonest age group affected was young adults in the prime working age groups. Though the number of cases with proven fungal isolates was in decreasing trend towards the elderly age group and younger age groups; accounting for around $14 \%$ in patients above 60 years of age and approximately $12 \%$ in patients below 20 years of age (Table 1).

Table 1: Distribution of fungal corneal ulcers in different gender and age groups

\begin{tabular}{|c|c|c|c|c|}
\hline $\begin{array}{l}\text { Age group } \\
\text { (Years) }\end{array}$ & Male & Female & Total & $\begin{array}{c}\text { Percentage } \\
\text { (\%) }\end{array}$ \\
\hline $0-10$ & 18 & 12 & 30 & 3.94 \\
\hline $11-20$ & 35 & 25 & 60 & 7.89 \\
\hline $21-30$ & 76 & 65 & 141 & 18.55 \\
\hline $31-40$ & 135 & 85 & 220 & 28.94 \\
\hline $41-50$ & 70 & 48 & 118 & 15.52 \\
\hline $51-60$ & 49 & 36 & 85 & 11.18 \\
\hline $61-70$ & 40 & 31 & 71 & 9.34 \\
\hline $71-80$ & 18 & 12 & 30 & 3.94 \\
\hline $81-90$ & 3 & 2 & 5 & 0.65 \\
\hline Total & $\begin{array}{c}444 \\
(58.42 \%)\end{array}$ & $\begin{array}{c}316 \\
(41.58 \%)\end{array}$ & 760 & 100 (99.55) \\
\hline
\end{tabular}

By occupation; more than half patients $51.18 \%$ of total fungal isolates were actively involved in agricultural work and farming followed by housewives $15.79 \%$, students $10.72 \%$ and other occupations (Table 2). 
Original Article | Bastola P et al. Pattern of fungal corneal isolates in mid western tertiary eye hospitals of Nepal

Table 2: Distribution of fungal isolates according to occupation of patients

\begin{tabular}{lcc}
\hline \multicolumn{1}{c}{ Occupation } & $\begin{array}{c}\text { Numberofpurefungal } \\
\text { isolates in patients }\end{array}$ & $\begin{array}{c}\text { Percentage } \\
(\%)\end{array}$ \\
\hline Agriculture(Farmers) & 389 & 51.18 \\
Housewives & 120 & 15.79 \\
Students & 80 & 10.52 \\
Office holders & 56 & 7.36 \\
Children & 35 & 4.60 \\
Labourers & 30 & 3.94 \\
Others & 50 & 6.57 \\
Total & 760 & 100 \\
\hline
\end{tabular}

Trauma or injury to cornea $431(56.71 \%)$, prior topical steroids use 89 (11.71\%), chronic ocular surface disorder 76 $(10.00 \%)$ were the most common predisposing risk factors noted which were followed by systemic diseases, contact lens wear and others (Table 3).

Table 3: Distribution of risk factors amongst the patients

\begin{tabular}{lcc}
\hline \multicolumn{1}{c}{ Risk factors } & $\begin{array}{c}\text { Number } \\
\text { of } \\
\text { cases }\end{array}$ & $\begin{array}{c}\text { Percentage } \\
(\%)\end{array}$ \\
\hline Trauma & 431 & 56.71 \\
Topical steroid use & 89 & 11.71 \\
Chronic ocular surface disorder & 76 & 10.00 \\
Systemic diseases & 54 & 7.10 \\
Contact lens wear & 52 & 6.84 \\
Others & 58 & 7.63 \\
Total & 760 & 100 \\
\hline
\end{tabular}

Fusarium species (Fusarium spp.) were the commonest fungal isolates accounting for 287 (37.76\%) of diagnosed fungal ulcer cases, followed by curvularia 136 (17.89\%), unidentified dematiaceous fungi 112 (14.73\%), aspergillus species $95(12.50 \%)$ and unidentified hyaline in 47 cases $(6.18 \%)$. While candida species, bipolaris species, penicillium, alternaria, mucor, rhizopus and unidentified were the other fungal isolates diagnosed (Table 4).
Table 4: Pure fungal isolates identified from patients with corneal ulcers

\begin{tabular}{|c|c|c|}
\hline Isolate & $\begin{array}{l}\text { Totalnumber } \\
\quad(n=760)\end{array}$ & $\begin{array}{c}\text { Percentage } \\
(\%)\end{array}$ \\
\hline Fusarium species & 287 & 37.76 \\
\hline Curvularia & 136 & 17.89 \\
\hline Aspergillus fumigatus & 47 & 6.18 \\
\hline Aspergillus flavus & 45 & 5.92 \\
\hline Aspergillus niger & 3 & 0.39 \\
\hline Unidentifieddematiaceousfungi & 112 & 14.73 \\
\hline Unidentified hyaline & 47 & 6.18 \\
\hline Candida albicans & 21 & 2.76 \\
\hline Candida tropicalis & 11 & 1.44 \\
\hline Candida parapsilosis & 3 & 0.39 \\
\hline Alterania species & 9 & 1.18 \\
\hline Bipolaris species & 8 & 1.05 \\
\hline Penicillium species & 8 & 1.05 \\
\hline Mucor & 4 & 0.52 \\
\hline Rhizopus & 3 & 0.39 \\
\hline Unidentified & 6 & 0.78 \\
\hline Others & 10 & 1.31 \\
\hline Total & 760 & $100(99.92)$ \\
\hline
\end{tabular}

\section{Discussion:}

A variety of factors determine clinical outcome in microbial (fungal and bacterial) keratitis. Epidemiological patterns may vary from country to country and between different geographical regions within a country. Corneal trauma and ulceration is the second leading cause of unilateral blindness. Corneal ulceration is seen in all ages. ${ }^{1}$

In this study, fungal corneal ulceration was more common in men $(58.42 \%)$, although earlier studies done in various parts and regions of India have reported a comparable gender distribution. ${ }^{13,21,22}$ Interestingly, in a study done in Kingdom of Bahrain ${ }^{23}$ the study has reported a higher incidence of corneal ulcer in women. ${ }^{23}$ The higher incidence rates seen in men in this study may be is due to the higher risk of exposure to injury during fieldwork/agricultural work and farming compared to women therefore developing more fungal corneal ulcers. Ulceration was more frequent among 
both men and women during the middle decades of life. Possibly, due to these age groups being maximally active outdoors. In this study too, the commonest occupation of the patients having proven fungal isolates were farmers which was followed by housewives, which correlates well with the occupational background.

The most common predisposing factor found in our study was corneal injury, accounting for $56.71 \%$ of total fungal isolates. This figure has been echoed by another study from south India. ${ }^{13}$ The agents responsible for this trauma were primarily agricultural matter such as rice stalks, thorns, tree branches and leaves and paddy grain. Animal products commonly implicated in corneal injury were the tails of cows and cow dung which consequently leads to develop a variable sized corneal injury leading to fungal corneal ulcers. Unlike in developed countries where causes such as the use of contact lens are reported to be common..$^{21,23}$ This is explained by the fact that most fungi isolated from the cultures of patients with corneal ulcer in this study are also commonly present as atmospheric saprophytes and can initiate an ulcer even with a small abrasion. Fungal corneal ulcer in contact lens users in our study accounted for a total of $6.84 \%$ of total fungal isolates which is quite less and correlates well with previous reports. ${ }^{21,23}$ Present study showed prevalence of chronic ocular surface disorder to be $10.00 \%$, prior topical steroid use $11.71 \%$ and underlying systemic diseases $7.10 \%$. Squamous blepharitis, meibomian gland dysfunction, chronic dacryosistitis were the reasons for ocular surface disorder becoming one of the very important risk factors to develop fungal corneal ulcer.

Of the systemic diseases Diabetes mellitus, immuno suppressive chronic systemic diseases, Hensen's disease, Rheumatoid arthritis, Tuberculosis contributed for $7.10 \%$ of risk factor for developing fungal corneal ulcers. Which did correlated well with a previous report from Nepal. ${ }^{24}$

The commonest fungus isolated in this study was fusarium species accounting for $37.76 \%$ of total fungal isolates, followed by curvularia species in $17.89 \%$ of cases, unidentified dematiaceous species in $14.73 \%$ of cases and aspergillus species in $12.50 \%$ of cases. Our study correlates well with a similar study done in Nepal earlier in the western peripheral terrain belt of Nepal and Indo-Nepal border ${ }^{24}$ Ghana and southern India. ${ }^{25}$ Where $32 \%$ and $39.9 \%$ of cases were due to fusarium species respectively. ${ }^{24,25}$ The fusarium species are common plant pathogens particularly of cereal crops and saprophytes of plant debris and are found in soil. The higher incidence of fusarium species may be due to the widespread cereal-crop cultivation in this region. ${ }^{24}$
Curvularia species were found to be more prevalent in our study $(17.89 \%)$ than in the study done in Ghana and Southern India in which the prevalence was $9.6 \%{ }^{22}$ In a similar study from $\mathrm{Nepa}^{24}$ curvularia species were found to be more prevalent, which correlated well with this study. ${ }^{24}$ In another study done in northern part of India, aspergillus species were found to be the most common fungal isolate accounting for $41 \%$ of cases. Though, in our study aspergillus species accounted for $12.50 \%$ of all pure fungal isolates. ${ }^{26}$ However few studies have shown aspergillus species to be the commonest fungal isolate isolated from patients with fungal corneal ulcer. ${ }^{21,22,27}$ This may be due to differences in the climate and natural environment of the areas studied.

This study showed high prevalence of unidentified dematiaceous and hyaline species. Dematiaceous species were diagnosed on the basis of the brown pigmentation similarly the hyaline species growth were identified as whites colonies.

Though, there has been already a report on culture positive fungal microbial keratitis from western terrain belt of Nepal, ${ }^{24}$ we believe the difference in geographical location and economic status plays a sufficiently important role to warrant the setup of our present study and helps in finding out the variation in the fungal isolates. Importantly, this study had more study subjects and more number of pure fungal isolates.

\section{Conclusion:}

In contrast to the other studies done in Nepal, this study found fusarium species to be the most common fungal isolate causing corneal ulcer followed by curvularia, unidentified dematiaceous fungi and aspergillus species. The higher incidence of fusarium species is contributed by the widespread cereal-crop cultivation in the study region. The study differed from various other studies primarily due to geographical location of the study population followed by occupation and socio economic status.

\section{Acknowledgements:}

We would like to thank all the patients, patient party and informant in helping us to carry out this study without their support this study would never have been completed. We would like to pass our debt to the microbiology team of Nepalgunj Eye Hospital and Nepalgunj Medical College for helping in providing accurate data, culture and sensitivity reports in time.

\section{Conflict of interest: None}




\section{References:}

1. Upadhyay MP, Karmacharya PC, Koirala S, et al. The Bhaktapur eye study: ocular trauma and antibiotic prophylaxis for the prevention of corneal ulceration in Nepal. Br J Ophthalmol 2001;85:388-392.

2. Chirambo MC, Tielsch JM, West KP, et al. Blindness and visual impairment in southern Malawi. Bull World Health Organ 1986;64:567-72.

3. Rapoza PA, West SK, Katala SJ, et al. Prevalence and causes of vision loss in central Tanzania. Int Ophthalmol 1991;15:123-9.

4. Brilliant LB, Pokhrel RP, Grasset NC, et al. Epidemiology of blindness in Nepal. Bull World Health Organ 1985;63:375-86.

5. Gilbert CE, Wood M, Waddel K, et al. Causes of childhood blindness in East Africa: results in 491 pupils attending 17 schools for the blind in Malawi, Kenya, and Uganda. Ophthal Epidemiol 1995;2:77-84.

6. Waddell K. Childhood blindness and low vision in Uganda. Eye 1998;12:184-92.

7. Smith GT, Taylor HR. Epidemiology of corneal blindness in developing countries. Refract Corneal Surg 1991;7:436-9

8. Yorston D, Foster A. Traditional eye medicines and corneal ulceration in Tanzania. J Trop Med Hyg 1994;97:211-4.

9. Whitcher JP, Srinivasan M. Corneal ulceration in the developing world: a silent epidemic. Br J Ophthalmol 1997;81:622-3.

10. Eric JC, Nevitt MP, Hodge DO, et al. Incidence of ulcerative keratitis in a defined population from 19501988. Arch Ophthalmol 1993;111:1665-71.

11. Gonzales CA, Srinivasan M, Whitcher JP, et al. Incidence of corneal ulceration in Madurai District, South India. Ophthal Epidemiol 1996;3:159-66.

12. Upadhyay MP, Karmacharya PC, Koirala S, et al. Epidemiologic characteristics, predisposing factors, and etiologic diagnosis of corneal ulceration in Nepal. Am J Ophthalmol 1991;111:92-9.

13. Srinivasan M, Gonzales CA, George C, et al. Epidemiology and aetiological diagnosis of corneal ulceration in Madurai, South India. Br J Ophthalmol 1997;81:965-71.

14. Bastola P. The pattern of Ocular morbidity, findings from a study conducted in western remote hilly region of Nepal. Nepal Journal of Medical Sciences. 2012;1:35-38.

15. Sutphen JE, Pelugfelder SP, Wilhelmus KR, et al. Penicillin Resistant Streptococcus Pneumoniae Keratitis. Am J Ophthalmol 1984;97:388-389.

16. Sonnenwirth AC, Jarett L. Gradwohl'S Clinical Laboratory And Diagnosis, 8Th Ed. Vol. Ii. U.S.A. Mosby, 1980.

17. Collee JG, Miles RS. Tests for Identification of Bacteria. In: Collee JG, Duguid JP, Fraser AG, Marmion BP. Mackie And Mccartney Practical Medical Microbiology, 13Th Ed. Vol. 2, New York: Churchill Livingstone, 1989: Pp. 456-481.

18. Thomas J, Liesegang TJ: Basic And Clinical Science Course; External Disease And Cornea, Section-8, American Academy of Ophthalmology, 2003.

19. Jones DB, Liesegang TJ, Robinson NM. Laboratory diagnosis of ocular infections. Washington DC: American Society for Microbiology, 1981.

20. Bharathi MJ, Ramakrishnan R, Vasu S, et al. Epidemiology of bacterial keratitis in a referral centre in south India. Indian J Med Microbiol 2003;21:239-45.

21. Basak SK, Basak S, Mohanta A, et al. Epidemiological and microbiological diagnosis of suppurative keratitis in Gangetic West Bengal, Eastern India. Indian J Opthalmol 2005;53:17-22.

22. Kumari N, Xess A, Shahi SK. A study of keratomycosis: our experience. Indian $\mathrm{J}$ Pathol Microbiol 2002; 45:299-302.

23. Al-Yousuf N. Microbial keratitis in Kingdom of Bahrain: clinical and microbiology study. Middle East Afr J Opthalmol 2009;16:3-7.

24. Ganguly S, Salma KC, Bastola P, et al. Pattern of fungal isolates in cases of corneal ulcer in the western periphery of Nepal. Nepal J Ophthalmol 2011;3:118-122

25. Leck AK, Thomas PA, Hagan M, et al. Aetiology of corneal ulceration in Ghana and South India and epidemiology of fungal keratitis. $\mathrm{Br} \mathrm{J}$ Ophthalmol 2002;86:1211-1215.

26. Chowdhary A, Singh K. Spectrum of fungal keratitis in North India. Cornea 2005;24:8-15.

27. Kotigadde S, Ballal M, Jyothirlatha, et al. Mycotic keratitis: a study in coastal Karnataka. Indian J Ophthal 1992;40:31-3 